\title{
A superlotação das maternidades em Pernambuco no contexto atual da política de saúde
}

\author{
Overcrowding in Pernambuco maternity in the context of current health policy
}

\author{
Marina Figueirêdo Assunção* \\ Raquel Cavalcante Soares** \\ Isadora Serrano*t.
}

\begin{abstract}
Resumo:
O presente estudo objetiva discutir a problemática da superlotação das maternidades públicas, no âmbito estatal, em suas mediações com as principais tendências da política de saúde atual, a partir da experiência da Emergência Obstétrica do Hospital das Clínicas de Pernambuco. A pesquisa consistiu em análise bibliográfica e documental - documentos e dados do Hospital das Clínicas de Pernambuco, notícias da mídia impressa que remetiam ao atendimento em Emergências Obstétricas no Brasil -, bem como de levantamento de dados estatísticos do Banco de Dados do Ministério da Saúde, referentes ao atendimento obstétrico no Estado de Pernambuco. A análise dos dados indicou um processo de intensificação da precarização do direito à saúde de qualidade (déficit de instalações físicas, falta de profissionais e leitos), e das condições de trabalho nas maternidades públicas, inclusive os impactos produzidos nas práticas em saúde. Dessa forma, concluímos que esse processo de precarização da saúde coloca grande obstáculo à efetivação da Política Nacional de Humanização do Parto e Nascimento, uma vez que viola diversos direitos das gestantes e dos recém-nascidos.
\end{abstract}

Palavras-chaves: Direito à saúde. Política de saúde. Serviços de saúde e humanização no parto.

\begin{abstract}
:
This study aims to discuss the problem of overcrowding in public hospitals within the state, in its mediations with the main trends of current health policy, taking as a starting point to the Emergency Obstetric Hospital of Pernambuco. The research was based on a survey of secondary data on the subject of databases of the Ministry of Health, University Hospital of Pernambuco and press reports regarding the number of beds, counseling, human resources and records of the Ombudsman, which referred to the service and internment in Emergency Obstetric the institution. The data analysis indicated an intensification process of casualization of the right to quality health (physical facilities, lack of professionals and beds), and working conditions in public hospitals. Precariousness that puts this large obstacle to the implementation
\end{abstract}

* Com a devida autorização da instituição, na forma de termo de consentimento. Assistente Social especialista em Saúde da Mulher pelo Programa de Residência Multiprofissional Integrada em Saúde do Hospital das Clínicas de Pernambuco. E-mail: marimariassuncao@hotmail.com

** Doutora em Serviço Social pela Universidade Federal de Pernambuco. E-mail: quelsoares@gmail.com

*** Mestre em Serviço Social pela Universidade Federal de Pernambuco. E-mail: isa sv@hotmail.com 
of the National Policy of Humanization of Childbirth, since it violates several rights of pregnant women and newborns.

Keywords: Right to health. Health policy. Health services e humanizing delivery.

\section{Introdução}

A problemática da superlotação das maternidades públicas no Brasil emerge com maior intensidade nos últimos anos. Tem se tornado frequente a publicização de denúncias - seja pelo movimento feminista, seja pelos trabalhadores do Sistema Único de Saúde (SUS), seja por representantes do poder público -, a respeito das dificuldades enfrentadas pelas parturientes nas maternidades públicas, principalmente no que se refere à indisponibilidade de leitos, de profissionais e instalações físicas precárias para atenção ao parto e nascimento.

De fato, usuárias e trabalhadores do Hospital Materno-Infantil Nossa Senhora de Nazareth (HMINSN), em Roraima, criticam a permanência das gestantes em poltronas nos corredores da unidade de saúde (LIMA, 2012); no Hospital Santa Mônica em Maceió/AL, "as mães e recém-nascidos permaneceram no chão da maternidade após parto" (SUPERLOTAÇÃO..., 2012); "por falta de vagas no Alojamento Conjunto e na Maternidade Barão de Lucena 25 mulheres foram admitidas em um pré-parto, onde a capacidade é para apenas dez parturientes" (ALMEIDA, 2012). Essas e outras notícias compõem a realidade da atenção ao parto e nascimento nas últimas décadas no país.

Apesar de tratar-se de uma problemática nacional, rebate desigualmente sobre as diferentes regiões e especialidades médicas conforme indica pesquisa realizada pelo Conselho Federal de Medicina (2012), a qual aponta uma redução de quase 42 mil leitos na rede pública de saúde, nos últimos sete anos. O Estado de Pernambuco, por exemplo, perdeu 1073 leitos, que correspondem a uma redução em 5,5\%.

A referida pesquisa destaca também que, dentre as especialidades mais atingidas com o corte, a obstetrícia aparece na terceira posição com uma redução de 5.862 leitos, ficando atrás apenas da psiquiatria 9.297 leitos e pediatria 8.979 (CONSELHO FEDERAL DE MEDICINA, 2012). Vale salientar que a redução dos leitos de psiquiatria refere-se ao processo de reforma antimanicomial. 
Já a Pesquisa de Assistência Médico-Sanitária (IBGE, 2010a) revelou que as regiões mais carentes de leitos por habitante são a Norte, com 1,8/1.000 leito, e Nordeste, com 2/1000 leitos, aquém da média estabelecida pelo Ministério da Saúde (BRASIL, 2002), que é de 2,5 a 3/1000 leitos hospitalares.

O número de leitos por mil habitantes estabelecido como o adequado pelo Ministério Saúde é significativamente menor que o de países como a Espanha, o qual apresenta um sistema de saúde semelhante ao brasileiro, porém dispõe de 4/1000 leitos. Além disso, destacamos que, apesar dessa definição do Ministério da Saúde, a média nacional brasileira é de 1,85/1000 leito (CONSELHO FEDERAL DE MEDICINA, 2012).

Esse déficit de leitos não se restringe aos serviços de Emergência Obstétrica, mas também aos demais que se encontram no âmbito da atenção materno-infantil. Em especial, os setores que se situam no atendimento ao parto e nascimento de alto risco, quais sejam: as Emergências Obstétricas, as Unidades de Cuidados Intermediários neonatais (UCIs), as Unidades de Terapia Intensiva Neonatal (UTIs), Alojamentos Maternos e os Alojamentos Conjuntos.

No cotidiano desses serviços, a insuficiente oferta de leitos, de profissionais e materiais, entre outras, intensifica situações como a peregrinação das parturientes das maternidades locais para interioranas ou o contrário; a negação do direito ao acompanhante no parto por falta de estrutura para acomodação; a restrição do acompanhante masculino pela inviabilidade de garantia da privacidade às parturientes; internamentos de gestantes de alto risco em macas, cadeiras de praia ou no chão, pela falta de leitos; a realização de transferências de gestações de alto risco pela ausência de material ou profissional para realizá-lo; a separação mãe e bebê após o parto pela indisponibilidade de leito em Alojamento Conjunto. Esses são exemplos de situações que compõem a rotina de maternidades públicas do Brasil.

O atual cenário da Política de Saúde expressa a violação do direito das parturientes à saúde de qualidade e humanizada. Direito esse, respaldado na Lei Orgânica da Saúde (LOS) 9.090/1988, na Política de Atenção Integral à Saúde das Mulheres (PAISM), no Programa de Humanização do Pré-Natal e Nascimento (PHPN), na Política Nacional de Atenção Obstétrica e Neonatal (PNAON) e no mais recente Programa Rede Cegonha. 
É importante considerar que, ao mesmo tempo que se dispõe de políticas que fundamentam e redirecionam o modelo de atenção à saúde na perspectiva da qualidade e humanização, a realidade demonstra a não efetivação de tais preceitos, expressa nas diversas dificuldades já explicitadas.

A inviabilidade de materialização desses direitos relaciona-se com as tendências da atual política de ajuste estrutural do Estado, política esta que se configura como estratégia do capital para enfrentamento à sua crise estrutural. Nesse sentido, no Brasil, são implementadas reformas desde a década de 1990 com a perspectiva de reduzir gastos sociais, que atacam o "caráter universal e público visando ao seu desmonte através de um processo de universalização excludente, mercantilização e privatização da saúde" (CORREIA, 2007, p. 1). Desse modo, observa-se que o processo de implementação do SUS, desde o início, materializou-se com limitações em seus princípios e diretrizes originais.

Esse processo contraditório expressou o tensionamento entre dois projetos antagônicos para a saúde, denominados por Bravo e Matos (2002) como projeto articulado ao mercado ou privatista e de reforma sanitária.

Importante situar que os problemas acima referidos não são inéditos, mas intensificados no atual contexto da Política de Saúde, de modo que as dificuldades vivenciadas, na atualidade, pelas parturientes já foram denunciadas, anteriormente, bem como objeto de enfrentamento de políticas e programas já construídos.

Sendo assim, a realidade da assistência à mulher parturiente, muitas vezes, caracteriza-se pela inadequação da assistência e outras situações injustas que contrariam a concepção de humanização e terminam por violar direitos estabelecidos.

Situações estas que também se fazem presentes na Emergência Obstétrica do Hospital das Clínicas de Pernambuco e que, no decorrer da vivência enquanto assistente social residente do Programa de Residência Multiprofissional Integrada em Saúde, suscitaram as seguintes questões: a superlotação seria uma questão específica da Emergência Obstétrica do Hospital das Clínicas de Pernambuco (HC/UFPE) ou de uma problemática transversal às maternidades públicas? Quais as mediações da superlotação e da humanização das maternidades com as tendências gerais da política de saúde atual, dentre elas a privatização da saúde pública? 
Nesse sentido, o presente artigo objetiva discutir a problemática da superlotação das maternidades do Sistema de Único de Saúde em Pernambuco, em suas mediações com as principais tendências da política de saúde atual, a partir da Emergência Obstétrica do Hospital das Clínicas de Pernambuco (EO-HC/UFPE).

Para isso, foi utilizada como estratégia de pesquisa a análise bibliográfica e documental, além de levantamento de dados secundários sobre a temática. A escolha da bibliografia se deu a partir das palavras-chaves: Direito à Saúde, Humanização no parto, Serviços de Saúde e Serviço Social na Saúde. Já a escolha dos documentos consistiu inicialmente do levantamento de notícias da mídia escrita - jornais nacional e local relativas ao atendimento de saúde materno-infantil no âmbito do SUS, e em seguida foram levantados documentos disponíveis que retratavam a realidade do HC/UFPE relatórios do Núcleo de Admissão e Alta da EO/HC; relatórios da Gestão de Pessoas, registros da Ouvidoria e da Direção do hospital. Os dados secundários foram obtidos no banco de Dados do Ministério da Saúde - DATASUS e Rede Interagencial de informações para Saúde (RIPSA).

A análise dos dados fundamentou-se na Teoria Social Crítica, ${ }^{1}$ uma vez que a interpretação dos dados pautou-se em uma compreensão de totalidade, "totalidade esta que buscou incorporar o movimento das contradições na realidade objetiva e aproximarse da essência do objeto de estudo, assim tornando-o consciente, em seus fundamentos, seus condicionamentos e seus limites" (PAULO NETTO, 2009).

\section{A contrarreforma da política de saúde no Brasil}

A Saúde tornou-se um direito de todos e dever do Estado, no Brasil, no contexto de redemocratização política, ou seja, no período de transição de um regime ditatorial para uma situação de estabilidade democrática, mesmo com o forte tensionamento entre o projeto de saúde privatista e o projeto sanitarista. ${ }^{2}$

\footnotetext{
${ }^{1}$ A teoria é, para Marx, a reprodução ideal do movimento real do objeto pelo sujeito que pesquisa: pela teoria, o sujeito reproduz em seu pensamento a estrutura e a dinâmica do objeto que pesquisa. E essa reprodução (que constitui propriamente o conhecimento teórico) será tanto mais correta e verdadeira quanto mais fiel o sujeito for ao objeto (NETTO, 2009).

2 Sobre projetos de reforma para a saúde ver Bravo e Matos (2002).
} 
A construção propriamente do Sistema Único de Saúde teve como marco inicial as proposições da VIII Conferência Nacional de Saúde, em 1986, momento em que os princípios e diretrizes do SUS foram sistematizados e amplamente discutidos. Grande parte dessas proposições foi legitimada pela Constituição Federal de 1988. Todavia, apenas em 1990, com a Lei 8080/1990 e 8142/1990, o Sistema Único de Saúde (SUS) foi regulamentado. Contraditoriamente, nessa mesma década, iniciou-se o processo de desmonte das políticas públicas, denominado de contrarreforma neoliberal.

Vale esclarecer que a opção pelo termo contrarreforma do Estado, ao processo reconhecido como reforma do Estado, comunga do entendimento de Behring (2003), a qual faz a reflexão de que tais mudanças operadas pelo Estado, na verdade, constituem-se em perda e desmonte dos direitos sociais, ao invés do fortalecimento e/ou ampliação desses como sugere o conceito de reforma. Os cortes dos gastos com as políticas sociais, via desmonte e precarização, são caracterizados como alternativa para o restabelecimento dos lucros do grande capital.

Nesse sentido, segundo afirma Behring e Boschetti (2007), evidencia-se a consequente desvinculação entre política social e direitos sociais. De modo que, nesse cenário, as políticas sociais assumem um caráter de focalização/seletividade, privatização e descentralização, perdendo, por sua vez, a perspectiva de universalidade.

Ainda em relação às características assumidas pela contrarreforma neoliberal na saúde, chama atenção a coincidente semelhança entre as orientações e características sinalizadas pelo Banco Mundial e as adotadas pelo Ministério da Saúde. Semelhanças estas que revelam, na verdade, a forte interferência desses organismos internacionais sobre os demais países.

\footnotetext{
As principais orientações do Banco Mundial (2005) sobre as denominadas "boas" políticas são: focalização seletiva no atendimento à pobreza, descentralização com responsabilização local na execução e financiamento das políticas com crescente desresponsabilização do nível central, responsabilização da sociedade civil com desresponsabilização do Estado, privatização/ mercantilização dos serviços (BANCO MUNDIAL, 2005, apud SOARES, R. 2007, p. $5)$.
}

De acordo com Correia (2007), essas "boas políticas" expressam as tendências da contrarreforma na saúde: via flexibilização da gestão dentro da lógica custo/benefício, do 
estímulo à ampliação do setor privado na oferta de serviços de saúde e do rompimento com o caráter universal do sistema público de saúde.

O discurso propagado é o de crise na saúde, portanto de inviabilidade de efetivação do SUS original e da necessidade de implementação de um SUS possível, diante do orçamento restrito, conforme denomina Soares, R. (2010). Em relação às manobras utilizadas para atacar e desconstruir o setor público, Simionatto $(2000$, p. 1) afirma que:

Inepto, ineficaz, ineficiente, responsável pelo déficit público são alguns dos adjetivos mais comuns que integram o discurso para designar a crise estrutural do Estado e cimentar a cultura que distorce e destrói a necessidade de sua existência. É no interior desse discurso que vem se fortalecendo a dicotomia entre "público" e "privado", caracterizando-se por público tudo o que é ineficiente, aberto ao desperdício e à corrupção, e por privado a esfera da eficiência e da qualidade.

Nesse sentido, Soares, R. (2010) afirma que, para o mercado poder ampliar sua inserção, torna-se necessário que a política de saúde pública seja precarizada. Esse processo de precarização se expressa na dificuldade do acesso à saúde, nas filas de espera, na baixa qualidade dos atendimentos prestados, na deterioração da estrutura física dos postos de atendimento e hospitais, na ausência de recursos materiais, na falta de contratação de profissionais, na flexibilização dos direitos dos trabalhadores que implica prejuízos na qualidade da assistência aos usuários, e da organização e luta desses trabalhadores (MARCH, 2011).

Contrapondo-se aos argumentos expostos pelo Banco Mundial e Ministério da Saúde, Correia (2007) demonstra que as principais causas que justificam a crise na saúde e, portanto, a não materialização do SUS original, estão relacionadas à precarização do trabalho e ao desfinanciamento ou subfinanciamento.

O Subfinanciamento se expressa na:

[...] diminuição progressiva do financiamento público da saúde, em especial no nível federal, com retração progressiva. [...]Ocasiona um ônus real para os mais frágeis, ou seja, os municípios e milhões de famílias brasileiras. É impossível um efetivo sistema público de saúde, universal e integral com o atual gasto anual per capita de US\$340, quando o gasto público per capita em sistemas europeus com diretrizes similares é de pelo menos US\$ 1000 por habitante (CENTRO BRASILEIRO DE ESTUDOS DE SAÚDE, 2011, p. 2). 
Tal realidade não significa que não há um crescimento do SUS, mas há uma ampliação restrita do Sistema, pois, conforme Soares, R. (2010, p. 56), "o sistema cresce, mas de forma precarizada e fragmentada e, sobretudo, não respondendo às reais necessidades da população". Diante disso, o perfil dos serviços de saúde no país reproduz, segundo Cohn, A. (1996, p. 26-28), as seguintes características:

\footnotetext{
Um SUS altamente centralizado, acentuadamente privatizado, crescentemente distante das reais necessidades de saúde da população brasileira, com clara divisão entre os trabalhadores entre setores públicos e privados, profundamente discriminatório e injusto e com acentuada distorção em sua forma de financiamento.
}

A precarização dos serviços de saúde, via subfinanciamento, conduz parcela da população a contratar planos privados de saúde. O relatório de Indicadores e Dados Básicos da Saúde, relativo à cobertura, demonstra essa tendência de crescimento, nos últimos quatro anos (de 2006 a 2009) da população coberta por planos privados de saúde, um aumento de 19,13\% 0 (BRASIL, 2010b).

As alternativas adotadas pelo Estado para enfrentamento da crise na saúde demonstram a intensificação da precarização do atendimento hospitalar, direcionada para a população pobre, induzindo àqueles que podem pagar a contratar planos privados de saúde de todos os tipos, em busca de melhor qualidade de atendimento. Isso demonstra que a Política de Saúde pública operacionalizada tem reproduzido hegemonicamente a lógica dos interesses do mercado, tanto no que se refere à privatização dos serviços públicos quanto no que se refere à sua precarização.

Sendo assim, o problema não se localiza na política original, porém, na forma como ela vem se efetivando para atender às necessidades do capital, em detrimento das necessidades da população. Essas tendências da política de saúde, de ampliação da privatização e precarização da saúde, incidem de modo particular sobre o campo de atenção à saúde da mulher.

\section{A política de atenção integral à saúde da mulher, o programa de humanização do pré-natal e nascimento e suas mediações com a contrarreforma do Estado}


A discussão das tendências da contrarreforma na política de saúde e suas mediações com o PHPN e com a PAISM torna-se central para a compreensão da superlotação das maternidades públicas nos últimos anos.

O sucateamento e a precarização da política de saúde incidem negativamente também sobre as políticas referentes à saúde das mulheres, que se expressa na negação do acesso aos serviços, nas filas de espera, na insuficiência do quadro de profissionais, na falta de estrutura física e insumos, no âmbito da atenção as parturientes. O caráter focalizado e pontual, que a política de saúde assume em tempos de contrarreforma neoliberal, contribui para a intensificação de violações de direitos que tangem à humanização e qualidade na atenção ao parto e nascimento.

A discussão em torno da humanização e qualidade na atenção às parturientes precisa ser realizada situando historicamente a construção do modelo de atenção ao parto no Brasil. O modelo de atenção ao parto hospitalar hegemônico, em muitos países, foi alvo de diversas denúncias, especialmente na década de 1980, no contexto de redimensionamento do próprio conceito de saúde e do movimento de reforma sanitária. Tais denúncias suscitaram a necessidade de criação de um novo modelo assistencial. É justamente nesse cenário que o campo da humanização e qualidade da atenção ao parto ganha destaque na cena pública.

Observa-se que paralelamente ao processo de contrarreforma neoliberal, desde a década de 1990, algumas importantes conquistas no campo da saúde das mulheres e da humanização na atenção ao parto merecem ser destacadas nesse período: o Programa de Humanização do Pré-natal e Nascimento (2000), a Política Nacional de Humanização da Gestão e Atenção (2003) e a Política Nacional de Atenção Integral à Saúde das Mulheres PAISM (2004).

No que se refere especificamente ao conceito de humanização na assistência ao parto, para Dias e Domingues (2005), alguns aspectos precisam ser considerados, tais quais: mudança na cultura hospitalar, com a organização de uma assistência realmente voltada às necessidades das mulheres e suas famílias; mudanças físico-estruturais para ambiente mais acolhedor; respeito pelos profissionais ao processo fisiológico; reconhecimento dos aspectos sociais e culturais presentes no parto e nascimento; a 
garantia de um acompanhante de sua escolha; o acesso à informação sobre todos os procedimentos a que serão submetidas; e dos seus direitos de cidadania respeitados.

$\mathrm{Na}$ perspectiva de nortear a implementação desse novo modelo assistencial, consolidou-se o Programa de Humanização do Pré-natal e Nascimento, o qual foi instituído pelo Ministério da Saúde, por meio da Portaria/GM n 569, de 1/6/2000, pautado nas análises das necessidades de atenção específica à gestante, ao recémnascido e à mãe no período do pré-natal e pós-parto (BRASIL, 2000a).

Vale sinalizar que esse programa reconhece que o atendimento digno e de qualidade são direitos inalienáveis de cidadania. De modo que, entre as metas do PHP, está o amparo das mulheres e recém-nascidos evitando a peregrinação em busca de atendimento; ampliar o acesso e qualidade do atendimento ao pré-natal e do pós-parto; intensificação de ações de planejamento familiar; além do investimento em sistemas de informação para obtenção de dados sobre óbitos de recém-nascidos e mães.

O conceito de humanização utilizado pelo PHPN considera dois aspectos fundamentais: um relativo ao tratamento digno pelas unidades de saúde e outro referente à adoção de medidas e procedimentos benéficos do acompanhamento do parto, evitando práticas intervencionistas desnecessárias.

Ainda relativo às conquistas, a Política de Atenção Integral à Saúde da Mulher PAISM é fruto dos esforços do movimento de mulheres e feminista para a elaboração de uma política que contemplasse as necessidades de saúde das mulheres, isto é, centrada na atenção integral à saúde, rompendo com o tradicional enfoque no período maternoinfantil. Desse modo, reafirma também os princípios norteadores da reforma sanitária, a ideia da descentralização, hierarquização, regionalização, equidade na atenção, bem como da participação social.

Apesar dos avanços, Silva e Reis $(2010$, p. 2) refletem ainda sobre os limites da perspectiva da integralidade na PAISM, quando indicam que:

A grande maioria das ações e estratégias propostas está relacionada à saúde sexual e reprodutiva, com ações voltadas para a gravidez e para prevenção das DST/HIV, não considerando as questões de gênero em sentido amplo, o que implicaria tomar como referência o modo como homens e mulheres se relacionam no seu cotidiano, enquanto resultado da determinação social do processo saúde/doença. 
Nesse sentido, a atenção integral à saúde ainda se coloca como um desafio para os serviços de saúde, tendo em vista a continuidade da ênfase no modelo materno-infantil e as diversas contradições no cotidiano de execução da política, perpassada pelas tendências da contrarreforma.

A Portaria 1.067/GM de 2005 que institui a Política Nacional de Atenção Obstétrica e Neonatal referencia algumas práticas para assistência qualificada e humanizada à mulher no pré-parto e parto, quais sejam:

O uso do partograma para registro da evolução do trabalho de parto; o teste rápido anti-HIV, com consentimento verbal da mulher; oferta de líquido por via oral durante o trabalho de parto; o respeito à escolha da mulher sobre o local e a posição do parto; respeito ao direito da mulher à privacidade no local do parto; fornecimento às mulheres das informações e explicações que desejarem; permissão de liberdade de posição e movimento durante o trabalho de parto; estímulo posições não-supinas durante o trabalho de parto; oferta de métodos não invasivos e não farmacológicos para alívio da dor, como massagens, banhos e técnicas de relaxamento durante o trabalho de parto; execução de procedimentos pré-anestésicos e anestésicos, quando pertinente; promoção do uso restrito de episiotomia (somente com indicação precisa); implementação de manejo ativo do terceiro período do parto com administração profilática de ocitocina; verificação da contratilidade uterina; exame rotineiro da placenta e das membranas ovulares; avaliação do canal de parto: sangramento, lacerações; implementar normas de prevenção de infecção; garantia da presença de pediatra na sala de parto, sempre que possível, ou de profissional capacitado para prestar os cuidados ao recém-nascido; garantia da realização do exame VDRL na mãe em sangue periférico; garantia imunoglobulina anti-Rh às mulheres iso-imunizadas; e elaboração dos relatórios médicos/enfermagem e do registro de parto e das condições de nascimento do RN (BRASIL, 2005b, p. 1)

Além disso, existem outros instrumentos no campo da humanização do parto e nascimento, como a Lei 11.108/2005 (BRASIL, 2005a) que dispõe sobre o direito às parturientes ao acompanhante no parto e pós-parto no âmbito do SUS, e a Lei $11.634 / 2007$, que dispõe sobre o direito da gestante ao conhecimento e vinculação com a maternidade onde receberá assistência no âmbito do SUS (BRASIL, 2007). Direitos esses que vêm sendo descumpridos, integral ou parcialmente, pela maioria das maternidades, resultando na dificuldade de efetivação das recomendações para uma assistência de qualidade e humanizada ao parto e nascimento.

Discutiremos as particularidades desse processo a partir da problemática da superlotação na Emergência Obstétrica do Hospital das Clínicas de Pernambuco. 


\section{A realidade das maternidades de Pernambuco e da emergência obstétrica do HC/UFPE no contexto da contrarreforma na política de saúde}

A caracterização de alguns aspectos socioeconômicos, demográficos e sanitários do Estado de Pernambuco é necessária para a compreensão da problemática da superlotação dos leitos obstétricos. Segundo o Censo Demográfico de 2010, Pernambuco tem uma população de 8.796 .032 milhões de habitantes, sendo que 4.504 .566 são do sexo feminino, ou seja, 51,9\% da população (IBGE, 2010). Segundo dados dos Indicadores e Dados Básicos (BRASIL, 2010c), Pernambuco detém um Produto Interno Bruto (PIB) per capita de $\mathrm{R} \$ \mathbf{8 . 0 6 4 , 9 7}$, mas $54,86 \%$ das pessoas sobrevivem com renda menor que $1 / 2$ salário mínimo e $17,86 \%$ da população é analfabeta.

No âmbito da saúde dispomos de 1,41 médicos por 1.000 habitantes, quando o preconizado pela Portaria ministerial é 2,5 (BRASIL, 2002). O quantitativo de leitos também se encontra abaixo do preconizado, com 1,14 leitos SUS públicos e 1,04 leitos SUS privados por 1000 habitantes (BRASIL, 2010a). Tais dados confirmam a ampliação da participação privada no SUS, uma vez que o que deveria ser complementar, praticamente, equipara-se ao ofertado pela esfera pública e, sobretudo, revela a precariedade no acesso e assistência à saúde. Acesso este que termina sendo focalizado na população mais pobre, uma vez que a oferta de médicos e leitos não corresponde à necessidade de toda a população. Sendo assim, aqueles que dependem do SUS, para acessá-lo, enfrentam filas de espera e atendimento precarizado.

Outro aspecto preocupante, e que tem relação com as manifestações da expressão da questão social, é o elevado número de mortes maternas. No Brasil, no ano de 2009 foram notificados 72 casos de óbitos maternos a cada 100.000 nascidos vivos (BRASIL, 2011).

No que tange à realidade da atenção ao parto no estado, o Cadastro Nacional de Estabelecimentos de Saúde (BRASIL, 2012) demonstra que se dispõe hoje de 2.560 leitos obstétricos e 137 maternidades cadastradas, no âmbito do SUS, as quais se encontram distribuídas entre os municípios em diferentes níveis de complexidade. Desse quantitativo total do estado, na capital, ainda de acordo com fonte acima referenciada, dispomos de 452 leitos. Tais dados evidenciam uma oferta de leitos aquém da necessidade da população com alto nível de feminização e majoritariamente SUS dependente, se 
considerarmos a base de cálculos do Programa Rede Cegonha, o qual indica que $75 \%$ da população é SUS dependente. Sendo assim, 75\% da população feminina, com base nos dados do IBGE (2010), representam 33.784.245 da população feminina dependente do SUS.

As ações e serviços de atenção ao parto seguem a lógica da integralidade da rede de saúde, sendo organizada por níveis de complexidade, os quais estão classificados como serviços de atenção ao parto de Baixo e de Alto Risco. Os serviços de Baixo risco são indicados para as gestantes que não apresentam fatores de risco para a gestação, os quais são relativos à idade, à história reprodutiva, doença obstétrica e intercorrências clínicas (BRASIL, 2000b).

Tabela 1 - Serviços de atenção ao parto no Estado de Pernambuco.

\begin{tabular}{llll}
\hline Serviço de Atenção ao Parto & SUS & Não SUS $^{\mathbf{3}}$ & TOTAL \\
\hline Baixo risco & 31 & 6 & 37 \\
Centros de parto normal & 10 & 5 & 15 \\
Alto risco & 9 & 11 & 20 \\
\hline Total & $\mathbf{5 2}$ & $\mathbf{2 2}$ & $\mathbf{7 4}$ \\
\hline
\end{tabular}

Fonte: Adaptado de Brasil (2012b).

Em relação aos dados evidenciados na tabela, cabe primeiramente considerar a desproporção de serviços comparados à densidade demográfica do estado, bem como destacar o número reduzido de serviços de alto risco a esse.

Pode-se inferir que o número reduzido de serviços de atenção ao parto na esfera privada demonstra o baixo interesse do mercado, uma vez que essa não é uma das áreas de maior lucratividade da saúde como alguns procedimentos da ortopedia, oncologia etc.

Os dados abaixo, relativos ao número de leitos obstétricos de internamentos cirúrgicos disponíveis no estado, facilitam a compreensão sobre os problemas no acesso aos serviços de atenção ao parto. Os dados a seguir representam o somatório de leitos obstétricos cirúrgicos na alta complexidade, no período de um ano - de agosto de 2011 a julho de 2012.

\footnotetext{
${ }^{3}$ Quando a tabela apresenta a legenda não SUS, remete aos leitos que não se situam em serviços de saúde pública, ou seja, aqueles que prestam atendimento apenas para planos privados de saúde e ou clientes particulares.
} 
Tabela 2 - Leitos obstétricos de internamentos SUS.

\begin{tabular}{|c|c|c|c|c|c|c|c|c|c|c|c|c|}
\hline $\begin{array}{c}\text { Mês } \\
2011 / 2012\end{array}$ & Ago & Set & Out & Nov & Dez & Jan & Fev & Mar & Abr & Mai & Jun & Jul \\
\hline $\begin{array}{l}\text { Leitos } \\
\text { Obstétricos }\end{array}$ & 2397 & 2389 & 2443 & 2435 & 2477 & 2467 & 2472 & 2511 & 2543 & 2556 & 2578 & 2560 \\
\hline $\begin{array}{l}\text { Aumento } \\
\text { em } 1 \text { ano } \\
\text { (\%) }\end{array}$ & & & & & & & & & & & & 6,4 \% \\
\hline
\end{tabular}

Fonte: Adaptado de Brasil (2012a).

Apesar de os dados do DATASUS demonstrarem a ampliação de leitos no estado, a realidade dos serviços de emergência obstétricas demonstra a recorrência de atendimentos e internamentos além da capacidade física e humana disponível, o que evidencia a insuficiente oferta e consequente superlotação dos serviços de atenção ao parto e nascimento.

Matérias do caderno Mais Saúde do mês de julho de 2012 confirmam a insuficiente oferta de leitos, a consequente superlotação, bem como a precariedade da assistência nas maternidades de Pernambuco: "Alto risco à saúde materna na RMR e Mata Sul"; "Promotoria da Saúde quer inspeção no Cisam"; "Sindicato dos médicos quer intervenção do Ministério da Saúde na crise das maternidades"; "Nova crise nas maternidades de Pernambuco; Emergência Obstétrica do IMIP mais uma vez fechada"; "Lotação excessiva no Barão de Lucena: Mulher com nove meses de gestação não encontra vaga em Recife"; "Desrespeito na hora do parto; Pai não é visita quer ser acompanhante na hora do parto" (ALMEIDA, 2012).

Diante do exposto, observa-se que, apesar da ampliação de leitos, de 6,4 \% no último ano, essa não supriu a necessidade da população. Demonstrando assim que há um crescimento do sistema, mas de forma precarizada e fragmentada, sendo focalizado na pobreza e flexibilizando espaço para o mercado, sem atender às reais necessidades da população usuária, configurando o que Soares, R. (2010) denomina de ampliação restrita.

Importante destacar a rede de serviços de atenção ao parto de Recife, que também se encontra organizada em diferentes níveis de complexidade. Nesse sentido, as instituições que se situam na atenção à gestação de baixo risco são a Maternidade Professor Barros Lima (MPBL), a Policlínica e Maternidade Professor Arnaldo Marques (PMPAM) e a Maternidade Bandeira Filho (MBF). Já entre as que se configuram como 
centros de parto estão o Instituto de Medicina Integral Professor Fernando Figueira (IMIP) e o Hospital Agamenon Magalhães (HAM). Na atenção a gestantes de alto risco, encontram-se o Hospital das Clínicas da Universidade Federal de Pernambuco (HC/UFPE), o IMIP, HAM, Hospital Barão de Lucena (HBL) e o Centro Integrado de Saúde Amaury de Medeiros (CISAM) (ALMEIDA, 2012).

A tabela 3, a seguir, apresenta a rede de serviços nos diferentes níveis de complexidade disponíveis na capital do estado.

Tabela 3 - Rede de serviços de atenção ao parto de Recife

\begin{tabular}{|c|c|c|c|c|}
\hline \multirow{2}{*}{$\begin{array}{l}\text { Serviço de } \\
\text { Atenção ao Parto }\end{array}$} & \multirow[t]{2}{*}{ SUS } & \multicolumn{3}{|c|}{ Quantidades de leitos } \\
\hline & & $\begin{array}{c}\text { Salas de } \\
\text { Parto Normal }\end{array}$ & Pré-parto & $\begin{array}{l}\text { Obstétricos } \\
\text { cirúrgicos }\end{array}$ \\
\hline \multirow[t]{3}{*}{ Baixo risco } & MPBL & 3 & 1 & 46 \\
\hline & PMPAM & 1 & 4 & 29 \\
\hline & MBF & 2 & 12 & 26 \\
\hline \multirow{2}{*}{$\begin{array}{l}\text { Centros de parto } \\
\text { normal }\end{array}$} & IMIP & 6 & 14 & 76 \\
\hline & HAM & 2 & 12 & 60 \\
\hline \multirow[t]{5}{*}{ Alto risco } & $\mathrm{HC}$ & 1 & 7 & 7 \\
\hline & IMIP & 6 & 14 & 76 \\
\hline & HAM & 2 & 12 & 16 \\
\hline & HBL & 1 & 4 & 29 \\
\hline & CISAM & 4 & 14 & 36 \\
\hline \multicolumn{5}{|c|}{ Fonte: Adaptado de Brasil (2012b). } \\
\hline \multicolumn{5}{|c|}{ *MPBL- Maternidade Professor Barros Lima } \\
\hline \multicolumn{5}{|c|}{ *PMPAM - Policlínica e Maternidade Professor Arnaldo Marques } \\
\hline \multicolumn{5}{|c|}{ *MBF - Maternidade Bandeira Filho } \\
\hline \multicolumn{5}{|c|}{ *IMIP - Instituto de Medicina Integral de Pernambuco } \\
\hline \multicolumn{5}{|c|}{ *HAM - Hospital Agamenon Magalhães } \\
\hline \multicolumn{5}{|c|}{ *HC - Hospital das Clínicas UFPE } \\
\hline \multicolumn{5}{|c|}{ *HBL - Hospital Barão de Lucena } \\
\hline${ }^{*} \mathrm{CISAM}$ - Centro In & de Saúde & deiros & & \\
\hline
\end{tabular}

A centralização da maioria dos serviços de alta complexidade na capital reflete uma lacuna no que tange à descentralização da política. Essa concentração de serviços vai acarretar, dentre outras, na disputa pela vaga, na peregrinação pelas maternidades, no grande volume de demandas nos serviços da capital. A dificuldade das parturientes no acesso à saúde na hora do parto é revelada também em pesquisa de Domingues (2012, p. 11), a qual afirma que " $20 \%$ das parturientes tiveram que passar por mais de um lugar, e mais de $3 \%$ passaram por mais de dois lugares na hora do parto." Essa insegurança de não 
ter uma vaga para internação no momento do parto e ter que peregrinar em busca dessa é por si só uma condição desumana para a gestante, contrapondo-se às propostas no campo da humanização do parto.

Apesar de algumas iniciativas já terem sido pensadas na tentativa de concretizar o princípio da descentralização, como exemplo a NOB/1993, é importante considerar que tal proposta, na verdade, reproduz um modelo de atenção à saúde, conforme considera Soares, R. (2010), à racionalidade instrumental - hospitalocêntrica, assistencial, fragmentada e com uma rede de serviços precarizada e insuficiente em relação à demanda. A distribuição dos serviços de atenção ao parto é desigual mesmo entre os cinco municípios mais populosos do estado, cuja população é superior a 50.000 habitantes.

Tabela 4 - População/município x leitos obstétricos hospitalares

\begin{tabular}{lcccc}
\hline Município & População & $\begin{array}{c}\text { População SUS } \\
\text { dependente }\end{array}$ & $\begin{array}{c}\text { Leitos Obstétricos - } \\
\text { cirúrgicos/SUS }\end{array}$ & $\begin{array}{c}\text { Média Leitos/ } \\
\mathbf{1 0 0 0} \text { hab. }\end{array}$ \\
\hline Recife & 1.537 .704 & 1.153 .278 & 280 & 0.18 \\
Jaboatão & 644.620 & 483.465 & 16 & 0.02 \\
Olinda & 377.779 & 284.334 & - & - \\
Caruaru & 314.012 & 235.509 & 23 & 0.07 \\
Paulista & 300.466 & 253.495 & - & - \\
Petrolina & 293.962 & 219.972 & 17 & 0.06 \\
\hline
\end{tabular}

Fonte: Adaptado de IBGE (2010).

Os dados apresentados demonstram que o número de leitos obstétricos por habitantes está aquém da necessidade da população, conforme parâmetro de 0,28 leitos por 1.000 habitantes SUS dependentes, bem como pelo fato de ainda não ter sido estruturado leitos obstétricos cirúrgicos nos municípios com mais de 50.000 habitantes, como estabelecido no Programa Rede Cegonha. Nesse sentido, a superlotação nos municípios de Recife, Jaboatão e Petrolina tem, também, relação com a ausência de serviços nas cidades circunvizinhas. A tabela 5 retrata a caótica realidade de superlotação da atenção às gestantes de alto risco em Pernambuco. Por meio da capacidade instalada, ou seja, da quantidade de leitos existentes e da capacidade de ocupação, demonstra o número real de gestantes internadas. 
Tabela 5 - Capacidade de leitos pré-parto nas emergências obstétrico alto risco em Pernambuco

\begin{tabular}{|c|c|c|c|}
\hline $\begin{array}{l}\text { Hospitais de } \\
\text { referência }\end{array}$ & $\begin{array}{l}\text { Capacidade } \\
\text { Instalada }\end{array}$ & Capacidade Ocupação & Total Ocupação (\%) \\
\hline HBL & 6 & 23 & $383 \%$ \\
\hline HAM & 12 & 23 & $192 \%$ \\
\hline HC & 7 & 10 & $143 \%$ \\
\hline CISAM & 13 & 28 & $215 \%$ \\
\hline IMIP & 14 & 24 & $171 \%$ \\
\hline HDM & 14 & 11 & $79 \%$ \\
\hline HJN & 11 & 3 & $27 \%$ \\
\hline MG & 7 & 7 & $100 \%$ \\
\hline $\begin{array}{l}\text { Fonte: Pernamb } \\
\text { *HBL - Hospital } \\
\text { *HAM - Hospital } \\
\text { *HC - Hospital dz } \\
\text { *CISAM - Centro } \\
\text { *HDM - Hospital } \\
\text { *HJN - Hospital J } \\
\text { * MG - Maternid }\end{array}$ & ledeiros & & \\
\hline
\end{tabular}

Os dados acima confirmam a superlotação e sua relação com a atual política de saúde, uma vez que se faz presente em todos os serviços citados. A situação da superlotação, ou seja, a falta de leitos para internamentos convive com outros aspectos que a intensificam e/ou por essa são intensificados, quais sejam, a intensificação da sobrecarga de trabalho da equipe, a falta de materiais (insumos, medicamentos etc.), que representam em prejuízos na qualidade da assistência prestada.

O Consolidado de leitos dos Hospitais de Referência para Alto Risco em Obstetrícia da Secretaria de Saúde do Estado de PE, do mês de maio de 2011, também revelou as principais causas da solicitação de fechamento dos serviços. Além da superlotação, outros problemas da ordem de recursos humanos e estrutura física também foram citados com frequência, tais quais: a falta de profissionais obstetras, neonatologistas e anestesistas, falta de material para realizar cesariana, reforma de bloco cirúrgico, falta de medicação para anestesia, surto de varicela, gestantes em cadeiras e puérperas na expectação, entre outros.

Sendo assim, o sucateamento das maternidades públicas e sua superlotação também expressa sobrecarga sobre os profissionais da saúde, que, segundo confirma Simionatto (1997), lidam com a baixa remuneração, atraso de salários, ausência de infraestrutura (física, humana e material), entre outras, dificultando a oferta de um serviço de qualidade. 
Antes de apresentar com maior detalhamento a realidade da superlotação da Emergência Obstétrica do HC/UFPE, faz-se necessário caracterizar a instituição em questão. A referida emergência localiza-se em uma instituição de natureza de administração direta, que presta serviço público e integra a rede de atenção ao parto e nascimento, como referência para o atendimento às gestantes de alto risco, prestando, assim, atendimento tanto às parturientes de demanda espontânea como encaminhadas pela central de leitos obstétricos. No Centro Obstétrico do HC funciona a Emergência Obstétrica - classificação de risco, triagem médica e consultas de emergência - e o Centro Obstétrico de fato, isto é, o internamento com quatro leitos de expectação ou pré-parto, duas salas de parto e três leitos de pós-parto imediato.

Apesar de o setor concentrar o pré-parto, parto e pós-parto, esse dispõe apenas de uma equipe para todos os atendimentos. A equipe, por plantão, é composta por cinco médicos obstetras, dos quais um chefe do setor, dois médicos assistentes, dois médicos residentes e três acadêmicos de medicina; um médico neonatologista plantonista e um diarista, uma enfermeira diarista e uma plantonista e quatro auxiliares de enfermagem. Além desses profissionais, compõem a equipe: nutricionistas, assistente social e psicóloga.

A tabela 6 corresponde a uma sistematização do quantitativo total de atendimentos, ou seja, do somatório de todas as gestantes que passaram pela triagem obstétrica, bem como do total de internamentos mensais, realizados pela equipe da Emergência Obstétrica do HC no último ano.

Tabela 6 - Atendimentos realizados na Emergência Obstétrica do HC/UFPE

\begin{tabular}{|c|c|c|c|c|c|}
\hline \multirow[t]{2}{*}{ MESES } & \multicolumn{2}{|c|}{ INTERNAMENTOS } & \multicolumn{2}{|c|}{ TRIAGEM } & \multirow{2}{*}{$\begin{array}{c}\text { TOTAL DE } \\
\text { ATENDIMENTOS }\end{array}$} \\
\hline & № & (\%) & № & (\%) & \\
\hline Ago & 151 & $63 \%$ & 164 & $36,9 \%$ & 444 \\
\hline Set & 280 & $38,6 \%$ & 229 & $61,4 \%$ & 373 \\
\hline Out & 144 & $34,6 \%$ & 285 & $65,4 \%$ & 436 \\
\hline Dez & 273 & $56,7 \%$ & 208 & $43,3 \%$ & 481 \\
\hline Nov & 183 & $36,5 \%$ & 319 & $63,5 \%$ & 502 \\
\hline Jan & 235 & $44 \%$ & 299 & $66 \%$ & 534 \\
\hline Fev & 241 & $52,7 \%$ & 216 & $42,3 \%$ & 457 \\
\hline Mar & 318 & $58,7 \%$ & 224 & $41,3 \%$ & 542 \\
\hline Abr & 260 & $49,3 \%$ & 267 & $50,7 \%$ & 527 \\
\hline Mai & 249 & $49,4 \%$ & 255 & $50,6 \%$ & 504 \\
\hline Jun & 437 & $98,9 \%$ & 5 & $1,14 \%$ & 442 \\
\hline Jul & 282 & $62,9 \%$ & 166 & $37,1 \%$ & 448 \\
\hline
\end{tabular}

Fonte: Elaboração própria com base nos dados da Universidade Federal de Pernambuco (2012a; 2012b). 
Os dados evidenciam um elevado número de atendimentos que não demandam internamento, com $41,2 \%$, que se referem principalmente a dificuldades existentes no pré-natal e que rebatem na atenção hospitalar. Apesar da ampliação do acesso ao prénatal, hoje, segundo Domingues (2012, p. 11), "99\% das gestantes realizam apenas uma consulta, mas apenas um número reduzido, $10 \%$ chegam ao final da gestação com o mínimo de consultas exigidas no protocolo".

As lacunas existentes nas redes de atenção ao parto dos municípios (intensa fragilidade em termos de recursos humanos, físicos e materiais), somadas à falta de esclarecimento das gestantes sobre aspectos relativos ao trabalho de parto, também podem ser refletidos sobre os dados apresentados.

Tabela 7 - Atendimentos realizados na Emergência Obstétrica do HC/UFPE

\begin{tabular}{lccc}
\hline MÊS & $\begin{array}{c}\text { TOTAL DE NOVOS } \\
\text { INTERNAMENTOS }\end{array}$ & $\begin{array}{c}\text { MÉDIA NOVOS } \\
\text { INTERNAMENTOS/ }\end{array}$ & $\begin{array}{c}\text { TOTAL } \\
\text { OCUPAÇÃO/CAPACIDADE } \\
\text { INSTALADA (\%) }\end{array}$ \\
\hline AGOSTO & 280 & DIA & $128 \%$ \\
SETEMBRO & 144 & 9,3 & $\mathbf{6 6 \%}$ \\
OUTUBRO & 151 & $\mathbf{4 , 6}$ & $71 \%$ \\
NOVEMBRO & 273 & 5 & $128 \%$ \\
DEZEMBRO & 183 & 9,1 & $85 \%$ \\
JANEIRO & 235 & 6 & $110,7 \%$ \\
FEVEREIRO & 241 & 7,5 & $114,4 \%$ \\
MARÇO & 318 & 8,3 & $142,3 \%$ \\
ABRIL & 260 & 10,2 & $115 \%$ \\
MAIO & 249 & 8,7 & $114 \%$ \\
JUNHO & $\mathbf{4 3 7}$ & $\mathbf{8}$ & $\mathbf{2 0 0 , 9 \%}$ \\
JULHO & 282 & $\mathbf{1 4 , 6}$ & $128 \%$ \\
TOTAL & 3364 & 9 & $\mathbf{1 2 8 , 3 \%}$ \\
\hline
\end{tabular}

Fonte: Elaboração própria com base nos dados da Universidade Federal de Pernambuco (2012a; 2012b).

A situação de superlotação do referido serviço no último ano foi uma constante com uma média de ocupação que extrapolou em $28 \%$ a capacidade instalada e, entre os meses, ganha destaque o de junho, uma vez que o serviço chegou a uma ocupação de $200,85 \%$.

Importante salientar que, ao longo deste período apresentado na tabela 7, segundo os ofícios encaminhados pela Direção do HC à Central de Regulação do Parto, em todos os meses foi feita, pelo menos, uma solicitação de fechamento do setor, devido à lotação do COB ou Unidade Neonatal. Vale destacar que o número menor de atendimentos - no mês 
de setembro - decorreu do fechamento do serviço para a realização de parto, devido ao surto de infecção hospitalar na Unidade Neonatal e reforma para adequação do setor.

A situação de superlotação traz implicações diretas para qualidade da assistência. Nos documentos do HC/UFPE constam relatos de dificuldades de realizar avaliação inicial em tempo às gestantes de alto risco em trabalho de parto, falta de leito/maca para proceder a triagem, a reavaliação sistemática das expectantes é prejudicada, os recémnascidos de risco permanecem em unidade de terapia semi-intensiva por falta de vaga na intensiva, e com proporção de um técnico para 6 recém-nascidos em cuidados intermediários ou intensivos.

A intensificação e prejuízos na assistência às parturientes também se evidencia quando a razão médico/paciente no HC/UFPE é de 4 obstetras para 7 parturientes em leitos de pré-parto e pós-parto, e a realidade do setor indica uma média de aproximadamente 4 obstetras para 9,2 parturientes. Salientando que se trata de uma média, uma vez que houve mês em que essa razão foi de 4 médicos para 14,6 usuárias. Sem considerar os aproximados 7,3 atendimentos/dia da triagem que não decorreram em internamento, conforme tabela 7.

Diante dessa realidade, o cotidiano de superlotação configura a atenção à saúde como algo emergencial e de grande sobrecarga de trabalho para os profissionais de saúde. Tal situação impõe aos profissionais uma resposta rápida às múltiplas demandas que Ihes chegam, indicando que as práticas para assistência qualificada e humanizada à mulher no pré-parto, parto e pós-parto, recomendadas na Política de Atenção Obstétrica e Neonatal e demais documentos afins, têm grande dificuldade de se concretizar.

Esses profissionais se esforçam, mesmo sem as condições, para oferecer uma assistência com o mínimo de qualidade e humanização às parturientes. De modo que, nesse contexto, há uma tendência de seleção e escalonamento de prioridades nos procedimentos, os quais, muitas vezes, já possuem indicação imediata. Além de haver dificuldades de realização do monitoramento adequado das parturientes, conforme recomendação dos protocolos de segurança. Nesse contexto, vale destacar que a criatividade e a improvisação, por vezes, fazem parte da rotina dos profissionais para garantir às parturientes assistência à saúde. 
Outro dado importante sobre a superlotação é os registros da Ouvidoria do HC/UFPE, que, no período de julho de 2011 a julho de 2012, também evidenciam as inquietações da população usuária quanto à qualidade da assistência. Das 10 reclamações, três remeteram a situações de constrangimento durante o atendimento, duas de dificuldades de marcação de exames, duas de extravio de prontuário, uma referente à estrutura física, uma à falta de atendimento e uma à insatisfação com as normas do hospital.

Além das 10 queixas, dois elogios também foram registrados, ambos relativos à excelência na qualidade do atendimento (UNIVERSIDADE FEDERAL DE PERNAMBUCO, 2012c). De tais registro, pode-se inferir que, apesar da realidade caótica vivenciada cotidianamente, mesmo sendo importante os registros, ainda são poucas as pessoas que procuram esse canal institucional para relatar as dificuldades vivenciadas. Além disso, esse dado faz refletir sobre o grau de conformismo da população em relação ao atendimento precário prestado pelo hospital.

Importante ressaltar que a superlotação não é exclusiva do HC/UFPE. Nesse sentido, o Jornal Diário de Pernambuco, na secção mais Notícias, apresenta que "na Maternidade do CISAM, 34 mulheres dividiam a sala de parto onde a capacidade é de 12 leitos"; e no "Hospital Barão de Lucena, a falta de leitos aumentava a tensão de parturientes que já se encontram em trabalho de parto" (ENCONTRO..., 2011).

Variados são os determinantes para a realidade de lotação das maternidades do estado hoje, entre elas foram publicizadas pelos poderes públicos e movimentos sociais a carência de obstetras e neonatologistas nos municípios do interior do estado, bem como o fechamento e/ou reformas de maternidades, tais quais a de Olinda, Maternidade Brites de Albuquerque, e a de São Lourenço da Mata, Maternidade Petrolina Campos. O que representou a redução de 80 leitos no último ano.

A Maternidade Brites de Albuquerque, apesar de reaberta, não aparece ainda no cadastro nacional dos serviços de saúde especializados na atenção ao parto e nascimento - DATASUS. Além de não cadastrada, segundo alerta Gigi, militante do movimento feminista, "o atendimento está sendo realizado em condições de extrema precariedade" (ALMEIDA, 2012). 
Frente às inúmeras denúncias e as cobranças do Ministério Público de Pernambuco (MPPE), algumas iniciativas foram tomadas pelo poder público. No sentido de amenizar a grave situação em que se encontram os serviços de atenção ao parto no estado, discussões e propostas foram realizadas pelo poder público e movimentos sociais. Em abril de 2011, por exemplo, o Secretário da Saúde do Estado, Antônio Figueira, e demais secretários dos seis municípios da Região Metropolitana do Recife - Olinda, Recife, Jaboatão, Camaragibe, Paulista e São Lourenço da Mata etc. - pactuaram a abertura, até o final do mês, de 20 leitos no Hospital de Jaboatão em Prazeres.

Em junho de 2011, o MPPE promoveu a Audiência Pública com representantes dos Secretários Municipais de Saúde do Estado, Secretaria Estadual de Saúde, Conselhos de profissões da área da saúde, representantes de diversas maternidades, sociedade civil, na tentativa de buscar medidas mais imediatas, como também reestruturadoras para área obstétrica (PERNAMBUCO, 2012b).

Em outra ocasião, o governador do estado, Eduardo Campos, lançou o Plano de Investimento em Assistência Obstétrica de Alto Risco, que prevê a construção de duas novas maternidades e a ampliação e descentralização dos serviços de obstetrícia em Pernambuco, em um prazo de dois anos. Conforme o plano, algumas ações estão previstas, quais sejam:

\footnotetext{
A requalificação de quatro unidades de baixo para alto risco; a construção duas novas maternidades, - Maternidade Metropolitana Sul de Alto Risco, em Jaboatão, e o Hospital da Mulher, em Caruaru - terão mais de 250 leitos, sendo 99 normais e 141 entre alto risco e UTI. A Maternidade Brites de Albuquerque será transformada na Maternidade Metropolitana Norte de Alto Risco, e o Hospital João Murilo, em Vitória de Santo Antão, se transformará também em uma maternidade de alto risco, assim como o Hospital Professor Agamenon Magalhães, em Serra Talhada. Também está prevista a ampliação do hospital Dom Malan, em Petrolina, e a intenção do governo é desafogar as maternidades da capital, acabar de vez com a superlotação dessas unidades e prestar um bom atendimento às mulheres grávidas que precisam realizar partos. $O$ investimento total, segundo o governador Eduardo Campos, é de R\$ 78 milhões (BRAGA, 2012, p. 1)
}

É importante ressaltar, no entanto, que, apesar da realidade da atenção maternoinfantil no estado encontrar-se caótica, a prioridade de investimento do governo de Pernambuco foi sobre as Unidades de Pronto Atendimento (UPA) - as quais são administradas por Organizações Sociais (PERNAMBUCO, 2011). Essas prioridades de 
investimentos precisam ser consideradas, uma vez que refletem a direção da política de saúde.

Diante da realidade caótica em que se encontram as maternidades públicas, o que é possível oferecer, em geral, é uma assistência aligeirada e com pouca qualidade. Assim, Fontes $(2007$, p. 301) estabelece uma analogia entre o cenário emergencial caótico da política de saúde atual e um naufrágio, que pode ser também identificado no âmbito da problemática aqui estudada. Nas condições de um naufrágio:

\begin{abstract}
Compreende-se que a atuação imediata não leve em consideração questões mais gerais como a lotação do barco; as condições de segurança para os passageiros (e não para a companhia de seguros); a necessidade daquele trajeto; a própria necessidade daquele barco (por que construímos barcos desse tipo e viagens daquela modalidade?); a necessidade de uma seletividade (salvaremos primeiro as crianças? as mulheres? os negros? os adultos? os jovens? as grávidas? os velhos e a memória do processo?); quem terá acesso ao oxigênio e às boias?, etc.). Nas dramáticas condições, a reflexão mais geral fica suspensa pela emergência da situação (FONTES, 2007, p. 301).
\end{abstract}

Essa analogia torna mais clara a refuncionalização dos princípios do SUS, tendo em vista que, diante da situação emergencial configurada na superlotação dos leitos obstétricos, os princípios fundamentais do SUS e os direitos relativos à saúde da mulher tendem a ser flexibilizados e, por sua vez, não são negados explicitamente.

Essa refuncionalização - que compõe a contrarreforma na saúde - torna-se evidente na atenção à parturiente quando o perfil das gestantes usuárias do SUS é majoritariamente composto por mulheres pobres e negras, o que confirma uma lógica de universalidade que focaliza a pobreza e que, desse modo, distancia-se do preconizado no SUS original.

Outro aspecto relativo à refuncionalização diz respeito à descentralização que, na verdade, opera como uma desresponsabilização da União. Desresponsabilização expressa no financiamento, o qual tem sido de responsabilidade preponderantemente dos municípios, sem o devido reconhecimento das desigualdades no que se refere, por exemplo, à capacidade de arrecadação desses.

A desresponsabilização se reflete numa organização e execução precária dos serviços de atenção às parturientes, ou seja, nas lacunas na oferta dos serviços nas maternidades municipais. Essas maternidades com frequência não realizam os 
atendimentos às gestantes, inclusive, as de baixo risco por problemas relativos à falta de profissionais (obstetras, neonatologistas e anestesiologistas), e/ou de material para parto cesáreo, e/ou de ambulância para transferências das gestantes como também de recursos físicos para o internamento com dignidade, é expressão da desresponsabilização da união.

No que se refere à integralidade, esta vem sendo operacionalizada de forma fragmentada, uma vez que a referência e contrarreferência, em geral, não fazem parte no atual contexto da atenção à parturiente. Pode-se dizer que a inviabilidade desse princípio encontra limite quando, sobretudo gestantes de baixo risco, pela indisponibilidade de recursos humanos, físicos e/ou materiais, precisam peregrinar pelas maternidades em busca de atendimento e vaga, inclusive, em outras cidades. Observa-se o que Soares, R. (2010, p. 57) denominou de um processo de fragmentação e precarização da política de saúde.

Nesse contexto, as práticas que possibilitam o aligeiramento do processo do parto, na verdade, tendem a ser mais recorrentes, a atenção emergencial se torna constante e a precarização do atendimento às parturientes é tratada como inevitável. Assim como, os princípios do SUS de universalidade, integralidade, equidade, descentralização e participação social são refuncionalizados.

A realidade da superlotação, dessa forma, viola os direitos das mulheres gestantes, os quais sejam: o direito à acompanhante, direito à assistência de qualidade, à garantia de uma vaga para gestante e recém-nascido na hora do parto, a uma experiência tranquila e familiar do evento do parto, bem como da evolução de um trabalho de parto com menor número de condutas invasivas.

Assim, é importante destacar a necessária reflexão sobre as práticas sociais na saúde no atual contexto da política. Soares, R. (2010, p. 50) destaca que "num cotidiano da urgência, no qual não há tempo para refletir, no máximo se pensa de modo imediato na ação, a lógica institucional impulsiona os profissionais a circunscreverem-se ao imediato, ao pragmático e ao urgente".

O/A assistente social como parte da equipe de profissionais de saúde também vivencia os rebatimentos desse contexto contraditório da atenção às parturientes. Esse contexto de superlotação das maternidades produz demandas específicas que se tornam 
objeto de intervenção do Serviço Social, ao mesmo tempo que o processo de precarização limitará a prática dos/as Assistentes Sociais.

Como aponta o Conselho Federal de Serviço Social (2010) sobre a atuação do Serviço Social na saúde, muitas demandas são relativas a soluções quanto ao atendimento solicitadas pelas usuárias e familiares - agilizar a realização de exames, realizar transferência para serviços mais próximos da residência, bem como a (im)possibilidade de participar na escolha do parto; e reclamação com relação à qualidade do atendimento e/ou ao não atendimento, que muitas vezes não se efetiva com base nos preceitos da humanização, as quais são: as relações com a equipe devido a ausência ou ao pouco esclarecimento sobre o tratamento, falta de medicamentos e exames diagnósticos, precariedade das instalações físicas, tempo de espera para realizar triagem médica e o próprio parto etc.

As insatisfações e precariedade quanto ao atendimento às parturientes resultam também em demandas da equipe de saúde para o assistente social, principalmente quanto ao convencimento da usuária para não desistir do tratamento, e solucionar falta de condições sociais para a sua realização.

Outra importante demanda se refere ao tensionamento das usuárias e seus familiares para a garantia do direito ao acompanhante no pré-parto, parto e pós-parto de livre escolha da mulher. Aqui, o contexto caótico da superlotação e inadequações das instalações físicas estabelecem mediações com a continuidade de um modelo de atenção à saúde hospitalocêntrico e medicocentrado, persistindo o entendimento do evento do parto como exclusivo dos profissionais de saúde e resultando na violação desse direito. O/A assistente social é requisitado pelas usuárias e familiares a mediar junto aos profissionais de saúde a presença do acompanhante, ao mesmo tempo que, muitas vezes, a equipe e a própria instituição solicitam a passivização e conformação dos usuários da impossibilidade de garantir esse direito.

Nesse sentido, Soares, R. (2010, p. 113) chama a atenção para a necessidade de analisar essas demandas, posto que "se não forem refletidas mais profundamente, se não buscarem ultrapassar o imediatismo, tendem a reproduzir a racionalidade hegemônica", e por conseguinte "ao contrário de mobilizar usuários e profissionais em torno do direito à 
saúde, passiviza-os", em uma espécie de ciclo perverso no qual a precariedade da política justifica a violação do direito.

\section{Considerações finais}

A superlotação das maternidades em Pernambuco e, particularmente na Emergência Obstétrica do HC/UFPE, no contexto da atual política de saúde, expressa o descompromisso do Estado com a vida/saúde das mulheres e seus filhos, as quais necessitam peregrinar e esperar muito por uma assistência precária. Mesmo com os avanços e mudanças de paradigmas contidos nos documentos oficiais - programas, políticas e planos no campo da atenção ao parto e nascimento -, os quais são baseados na perspectiva da qualidade e humanização, a realidade sugere a intensificação da precariedade da assistência.

A política de saúde implementada e norteada majoritariamente pelos interesses do mercado repercute na atenção às parturientes de modo perverso, uma vez que a lógica da Política operacionalizada não viabiliza as condições para a garantia dos princípios da qualidade e humanização, bem como viola outros direitos relativos ao evento do parto e nascimento.

De fato, o que tem se verificado é a materialização de um modelo de atenção perpassado por diversas contradições, entre a afirmação e a negação do direito, que, muitas vezes, não se pauta na saúde de qualidade, porém se estrutura na lógica do custobenefício, na focalização da assistência e numa assistência de natureza emergencial e precarizada para as mulheres.

Nesse sentido, a realidade de superlotação na Emergência Obstétrica do HC/UFPE evidencia que a qualidade e humanização não vêm se materializando conforme os princípios originais do SUS e do projeto de reforma sanitária, em um cenário em que até o acesso é problemático. Todavia, dialeticamente, é nesse mesmo contexto em que o termo humanização vem ganhando maior espaço, mas num sentido hegemonicamente disseminado em que se transfere para os trabalhadores toda a responsabilidade por tal prática, desconsiderando as condições de trabalho, a estrutura e financiamento dos serviços, uma vez que a humanização no seu sentido amplo carece das condições físicas, materiais e humanas para se efetivar. 
O estudo demonstra a precariedade da assistência às gestantes e condições de trabalho dos profissionais. Usuárias que vivenciam o parto como experiência traumática e trabalhadores que prestam a assistência possível com os recursos disponíveis, muitas vezes, com o dobro do quantitativo da capacidade de atendimento.

Os desafios postos aos trabalhadores da saúde, em especial a/ao assistente social, por assumir o compromisso em defesa dos interesses da classe trabalhadora, para o enfrentamento dessa realidade é a construção de estratégias por meio do debate coletivo, da articulação dos profissionais e a reflexão crítica e aprofundada sobre as mediações que constitui esse contexto. Para tanto, faz-se necessário superar as práticas aligeiradas, imediatas e fragmentadas, que procure superar a simples indignação com o cotidiano de trabalho e se transforme em ação coletiva consciente das determinações presentes nesse processo.

Caso contrário, o possibilismo e a flexibilização dos princípios e direitos conquistados a partir da reforma sanitária continuarão a ser argumentos presentes e fortemente disseminados, que se traduzem na aceitação da realidade atual, dos baixos investimentos com a saúde e, assim, na aceitação da oferta de um serviço precário, pobre para pobres. Nessa perspectiva, não se faz relevante a qualidade, ou a qualidade assume uma perspectiva do custo-benefício associada a simples produtividade.

No entanto, importa destacar que contraditoriamente em meio à negação do direito ao parto humanizado, a afirmação do direito também se faz presente, em geral, na assistência à saúde prestada às parturientes. Desse modo, mesmo com todas as dificuldades e tendências adversas da contrarreforma, a maior parte das mulheres conseguem ter atendimento, ainda que emergencial. Aqui, particularmente, expressa-se o tensionamento entre os projetos societários no âmbito da política de saúde, na forma do projeto de reforma sanitária e dos projetos privatistas e do SUS possível.

Podemos concluir, assim, que a lógica predominante nesses serviços que sofrem intensamente a problemática da superlotação é a da contrarreforma e dos projetos privatistas e SUS possível, em que se pecariam as condições em que é prestada a assistência, ao sobrecarregarem os profissionais de saúde, impactando nas práticas de forma a torná-las tendenciadas ao imediatismo e pragmatismo. 


\section{Referências}

ALMEIDA, V. Alto risco à saúde materna na RMR e Mata Sul. Jornal do Comércio, São Paulo, 19 jul. 2012. Caderno Cidades. Disponível em:

$<$ http://jconlineblogs.ne10.uol.com.br/maissaude/alto-risco-a-saude-materna-na-rmr-emata-sul/>. Acesso em: 20 ago. 2012.

BEHRING, E. R. Brasil em contra-reforma: desestruturação do estado e perda de direitos. São Paulo: Cortez, 2003.

BEHRING, E. R.; BOSCHETTI, I. Política social: fundamentos e história. São Paulo: Cortez, 2007.

BRAGA, C. Mais leitos para partos de alto risco. Construção e ampliação de maternidades, anunciadas pelo governo, pretendem desafogar rede. Diário de Pernambuco, Pernambuco, 15 maio 2012. Disponível em: <http://www.hemope.pe.gov.br/ download/15052012_Clipping_Eletrnico_SES.pdf>. Acesso em: 12 jul. 2012.

BRASIL. Ministério da Saúde. Portaria no 569, de 1 de junho de 2000. Institui o programa de humanização no pré-natal e nascimento, no âmbito do Sistema Único de Saúde. Diário Oficial da União, Poder Executivo, Brasília, DF, 8 jun. 2000a.

Ministério da Saúde. Secretaria de Políticas Públicas. Gestação de alto risco. Brasília, 2000b. Manual técnico.

BRASIL. Ministério da Saúde. Portaria no 1101/GM, de 12 de junho de 2002. 2002. Institui os parâmetros assistenciais do SUS. Disponível em: <http://dtr2001.saude.gov.br/sas/ PORTARIAS/Port2002/Gm/GM-1101.htm>. Acesso em: 12 jul. 2012.

Lei no 11.108 , de 7 de abril de 2005. Altera a Lei no 8.080, de 19 de setembro de 1990 , para garantir às parturientes o direito à presença de acompanhante durante o trabalho de parto, parto e pós-parto imediato, no âmbito do Sistema Único de Saúde SUS. Diário Oficial da União, Poder Executivo, Brasília, DF, 8 abr. 2005a.

Portaria no 1.067/GM de 4 de julho de 2005. Institui a Política Nacional de Atenção Obstétrica e Neonatal, e dá outras providências. 2005b. Disponível em: <http://dtr2001.saude.gov.br/sas/PORTARIAS/Port2005/GM/GM-1067.htm>. Acesso em: 20 dez. 2005.

Lei no 11.634 , de 27 de dezembro de 2007. Dispõe sobre o direito da gestante ao conhecimento e a vinculação à maternidade onde receberá assistência no âmbito do Sistema Único de Saúde. Disponível em: <http://www.planalto.gov.br/ccivil_03/_Ato20072010/2007/Lei/L11634.htm>. Acesso em: 20 dez. 2012.

Ministério da Saúde. Cadastro Nacional de Estabelecimentos de Saúde - CNES. Indicadores de recursos: número de leitos hospitalares por habitante. 2010a. Disponível em: <http://tabnet.datasus.gov.br/cgi/tabcgi.exe? idb2010/e01.def>. Acesso em: 25 set. 2012. 
. Ministério da Saúde. Indicadores de cobertura: Cobertura de planos privados de saúde. 2010b. Disponível em: <http://tabnet.datasus.gov.br/cgi/tabcgi.exe?idb2010/ f16.def>. Acesso em: 20 set. 2012.

Ministério da Saúde. Indicadores Socioeconômicos: Produto Interno Bruto (PIB) per capita. 2010c. Disponível em: <http://tabnet.datasus.gov.br/cgi/tabcgi.exe? idb2010/b03.def>. Acesso em: 20 set. 2012.

Ministério da Saúde. Indicadores de Mortalidade: razão de mortalidade materna. 2011. Disponível em: <http://tabnet.datasus.gov.br/cgi/idb2011/C03b.htm>. Acesso em: 20 set. 2012.

Ministério da Saúde. Cadastro Nacional de Estabelecimentos de Saúde - CNES. Recursos físicos: leitos de internação - Brasil - Pernambuco, jul. 2012a. Disponível em: <http://tabnet.datasus.gov.br/cgi/tabcgi.exe?cnes/cnv/leiintbr.def>. Acesso em: 25 set. 2012.

Ministério da Saúde. Cadastro Nacional de Estabelecimentos de Saúde - CNES Serviços especializados: serviços de atenção ao pré-natal, parto e nascimento - parto em gestação de alto risco - Pernambuco. Disponível em: <http://cnes.datasus.gov.br/ Mod_Ind_Especialidades.asp?VEstado $=26 \& V M u n=\& V T e r c=00 \& V S e r v i c o=112 \&$ VClassifica cao $=004 \& V A m b u=\& V A m b u S U S=\& V H o s p=\& V H o s p S U S=1>$. Acesso em: 26 set. $2012 b$.

BRAVO, M. I. S.; MATOS, M. C. A saúde no Brasil: reforma sanitária e ofensiva neoliberal. In: BRAVO, M. I. S.; PEREIRA, P. A. P. (Org.). Política social e democracia. São Paulo: Cortez, 2002. p. 197-215.

CENTRO BRASILEIRO DE ESTUDOS DE SAÚDE - CEBES. Renovar a política: preservando o interesse público na saúde. Rio de Janeiro, nov. 2011. Disponível em:

<http://www.cebes.org.br/media/file/Renovar\%20a\%20

Politica\%20Preservando\%200\%20Interesse\%20Publico\%20na\%20Saude.pdf>. Acesso em: 18 ago. 2012.

COHN, Amélia. Desigualdade, desenvolvimento social e políticas sociais no Brasil. São Paulo: Cedec, 1996, 44p. (Cadernos Cedec, no 57).

CONSELHO FEDERAL DE MEDICINA. Crise na assistência: falta de financiamento impacta no número de leitos e prejudica trabalho médico. 2012. Disponível em:

$<$ http://portal.cfm.org.br/index.php?option=com_content\&view=article\&id=23234:crisena-assistencia-falta-de-financiamento-impacta-no-numero-de-leitos-e-prejudica-trabalhomedico\&catid=3>. Acesso em: 19 set. 2012.

CONSELHO FEDERAL DE SERVIÇO SOCIAL. Parâmetros para atuação de assistentes sociais na saúde. Brasília: CFESS, 2010.

CORREIA, M. V. C. A saúde no contexto da crise contemporânea do capital: o Banco Mundial e as tendências da contra-reforma na política de saúde brasileira. Temporalis, São Luiz, v. 7, n. 13, jan./jun. 2007. 
DIAS, M. A. B.; DOMINGUES, R. M. S. M. Desafios na implantação de uma política de humanização da assistência hospitalar ao parto. Ciência Saúde Coletiva, Rio de Janeiro, v. 10, n. 3, p. 669-705, set. 2005.

DOMINGUES, R. M. S. M. Pré-natal: alta cobertura, baixa eficiência. Radis, Rio de Janeiro, n. 117, maio, 2012.

ENCONTRO traça metas para abertura de novos leitos em maternidades. Diário de Pernambuco, Recife, 19 abr. 2011. Secção Saúde. Disponível em:

<http://www.old.diariodepernambuco.com.br/vidaurbana/nota.asp?materia=201104191 01411>. Acesso em: 10 jun. 2012.

FONTES, V. Equidade, Ética e Direito à Saúde. Estudos de politécnica e saúde: volume 2 / Organização da Escola Politécnica de Saúde Joaquim Venâncio. Coordenação de Isabel Brasil Pereira e Claudio Gomes Ribeiro. - Rio de Janeiro: EPSJV, 2007. Disponível em: <http://www.acervo.epsjv.fiocruz.br/beb/textocompleto/008942>. Acesso em 12.06.2012.

IBGE. Estatísticas de saúde. Assistência médico-sanitária 2009. Rio de Janeiro, 2010a. Disponível em: <http://www.ibge.gov.br/home/estatistica/populacao/condicaodevida/ ams/2009/ams2009.pdf>. Acesso em: 12 jun. 2012.

. Sinopse do Censo Demográfico 2010. 2010b. Disponível em:

<http://www.ibge.gov.br/home/estatistica/populacao/censo2010/tabelas_pdf/Brasil_tab _1_14.pdf>. Acesso em: 12 jun. 2012.

LIMA, V. Parturientes ficam nos corredores por falta de leitos na Maternidade. Jornal Folha Web, Boa Vista, 19 jan.2012. Disponível em: <http://www.folhabv.com.br/mobile/ noticia.php?id=122956>. Acesso em: 12 jul. 2012.

MARCH, C. A contrarreforma do estado e o trabalho em saúde nos serviços públicos: uma análise da realidade brasileira. Tempus - Atas de Saúde Coletiva, Brasília, v. 5, n. 1, p. 175189, 2011.

NORONHA, J. C.; SANTOS, I. S.; PEREIRA, T. R. Relações entre o SUS e a saúde suplementar: problemas e alternativas para o futuro do sistema universal. In: SANTOS, N., AMARANTE, P. D. C. (Org.). Gestão pública e relação público-privado na saúde. Rio de Janeiro: Cebes; 2010. Cap.9, p.152-179.

PAULO NETTO, J. Introdução ao método da teoria social. 2009. Disponível em: <http://pcb.org.br/portal/docs/int-metodo-teoria-social.pdf >. Acesso em: 12 nov. 2013.

PERNAMBUCO. Secretaria de Saúde. Consolidado de leitos dos hospitais de referência para alto risco em obstetrícia em PE. Recife, maio 2011.

Focos estratégicos: saúde. Disponível em: <http://www.pe.gov.br/governo/focosestrategicos/saude/>. Acesso em: 10 ago. 2012a. 
. Ministério Público Estadual. Ministério Público busca solução para superlotação das maternidades. Diário Oficial, Recife, n. 117, 23 jun. 2012b. Disponível em: $<$ http://siteantigo.mppe.mp.br/uploads/NNb73QcmMaSOBQPqw6EIGQ/jx4qDdP1xql9dYJAtpHaA/2011-06-23.PDF>. Acesso em: 20 set. 2012.

SILVA, T. C. F.; REIS, T. C. P. Serviço social e atenção integral à saúde da mulher. Considerações sobre a ação profissional no Serviço de Ginecologia do HFB. In: SEMINÁRIO REGIONAL DE ESTÁGIO E EXTENSÃO EM SERVIÇO SOCIAL, 2., 2010, Niterói.

Anais...Disponível em: <https://docs.google.com/viewer?a=v\&pid=sites\&srcid= ZGVmYXVsdGRvbWFpbnx0cmFiYWpvc29jaWFseXNhbHVkfGd4OjQ0Y2Q5OGVkYWZmZGM xZWQ>. Acesso em: 7 ago. 2012.

SIMIONATTO, I. Globalitarismo e sociedade civil: a manipulação dos conceitos. Florianópolis, 1997.

Reforma do Estado ou modernização conservadora? 2000. Disponível em:<http://www.acessa.com/gramsci/?id=56\&page=visualizar $>$. Acesso em: 10.08.2012.

SOARES, A. Trabalho, saúde e gênero. 2010. Disponível em:

$<$ http://angelapstu16123.blogspot.com/2010/08/trabalho-saude-e-genero.html>. Acesso em: 10 set. 2011.

SOARES, R. C. Mundialização do capital e contrarreforma do estado brasileiro: o importante papel ideopolítico do Banco Mundial. In: JORNADA INTERNACIONAL DE POLÍTICAS PÚBLICAS, 3., São Luís. Anais... São Luís, 2007.

A contrarreforma na política de saúde e o SUS hoje: impactos e demandas ao Serviço Social. 2010. Tese (Doutorado em Serviço Social) - Universidade Federal de Pernambuco, Recife, 2010.

SUPERLOTAÇÃO: pacientes são atendidas no chão da maternidade Santa Mônica.2012. Disponível em: <http://primeiraedicao.com.br/noticia/2012/07/23/superlotacaopacientes-sao-atendidas-no-chao-na-maternidade-santa-monica>. Acesso em: 26 set. 2012.

UNIVERSIDADE FEDERAL DE PERNAMBUCO. Hospital das Clínicas. Central de Regulação de partos. Ofícios Direção HC: jul./2011 a jul./2012. Recife, 2012a. Documento Interno.

. Hospital das Clínicas. Núcleo de Admissão e Alta do COB/HC-UFPE. Planilha de atendimentos e internamentos: jul./2011 a jul./2012. Recife, 2012b. Documento Interno.

. Hospital das Clínicas. Ouvidoria. Ficha de ocorrência e despacho: Finalidade reclamação (Relatório no período de 01.01.2011 à 30.07.2012). Recife, 2012c. p.1-3. Documento Interno.

Recebido em: 02/03/2013

Aprovado em: 11/02/2014 